\title{
Hybrid Technique: Common Femoral Artery Endarterectomy and Endovascular Therapy in Critical Lower Limb Ischemia
}

\author{
By \\ Ahmed Fekry ${ }^{1}$ Samer Regal ${ }^{1}$ Hosam Elwakil ${ }^{1}$ Ehab Saad ${ }^{1}$ \\ Vascular surgery department, Mansoura Faculty of Medicine
}

DOI : 10.21608/mimu.2021.66114.1011

Submit Date: 2021-03-03 Accept Date : 2021-03-18 Available online:

\section{Keywords}

- Hybrid

- Endarterectomy

- Endovascular

\begin{abstract}
Multilevel arterial occlusive disease in patients with chronic limb threatening ischemia (CLTI) represent a major revascularisation challenge to vascular surgeons. The goal of treatment is a single intervention procedure that results in an excellent technical success without associated complications or the need for further interventions. Methods: Prospective case series study including (30) patients complaining from critical lower limb ischemia (rest pain or tissue loss) and has CFA lesionwith proximal or distal lesions. All patients undergoing common femoral Endarterectomy and endovascular therapy to proximal or distal lesions between May 2018 and December 2020. The study was conducted at the department of vascular and endovascular surgery in Mansoura university hospitals. Results: Femoral Endarterectomy was performed in all cases in the current cohort with combined iliac and SFA revascularization in $27 \%$ of patients and with separate iliac in $10 \%$ and outflow in $63 \%$ of cases (SFA $33 \%$ and tibial $30 \%$ ). This study reported a $100 \%$ technical success and $96 \%$ hemodynamic and clinical success in the current series. And the 2-year patency was $88 \%$. Conclusion: CFE should remain the treatment of first choice of CFA occlusive disease as it is safe, effective, and durable procedure for common femoral artery disease, and the simultaneous hybrid CFA endarterectomy and endovascular therapy for multilevel occlusive artery disease can provide an immediate satisfactory results, durable midterm patency, and also less invasive therapeutic option in high-risk patients..
\end{abstract}

Corresponding author: Ahmed Fekry, Vascular surgery, faculty of medicine, mansoura university, E mail. dahmedfekry@gmail.com . Tel. 01002710565 


\section{INTRODUCTION}

Multilevel arterial occlusive disease in patients with chronic limb threatening ischemia (CLTI) represent a major revascularisation challenge to vascular surgeons. The goal of treatment is a single intervention procedure that results in an excellent technical success without associated complications or the need for further interventions. (1)

CLTI is usually not related to isolated aorto-iliac or superficial femoral artery (SFA) disease; tandem lesions involving the femoropopliteal combined with aorto-iliac or infrapopliteal segments are mostly present. Recent guidelines on the treatment of peripheral arterial occlusive disease recommend an endovascular procedure as a first strategy in treating short arterial occlusions, and accept that indication in extensive lesions in high risk patients, or when conventional surgical options are not possible. However, common femoral artery (CFA) disease remains an area of surgical priority.(2)

Common femoral artery endarterectomy (CFE) is still considered to be the preferred surgical technique to treat common femoral artery lesions. These lesions are bulky, eccentric, and calcified, often involve the origin of the profunda femoris artery that is recognized as a critical source of collateral vessels. They are also in an area of high mobility, and the repair would not be expected to fare well after stent placement. CFE exhibited a high technical success rate of $85-100 \%$ as well as low morbidity and mortality rates of 6.6$22 \%$ and $0-2 \%$, respectively.In addition to low morbidity and mortality rates, long-term outcomes after isolated CFE are also favorable. (3)
The current prospective study aimed to assess the outcomes of common femoral endarterectomy combined with an inflow and outflow endovascular revascularization procedure as an alternative for high risk patients with CLTI. Included cases were treated rather homogenously under local or regional anaesthesia.

\section{Patients and methods:}

\section{Study Design}

\section{Prospective case series study including} (30) patients complaining from critical lower limb ischemia (rest pain or tissue loss) and has CFA lesion (tight stenosis $\geq 50 \%$ or total occlusion) with proximal (iliac) or distal (femoropopliteal or tibial) lesions

All patients undergoing common femoral Endarterectomy and endovascular therapy to proximal or distal lesions between May 2018 and December 2020. The study was conducted at the department of vascular and endovascular surgery in Mansoura university hospitals.

\section{Patient evaluation}

Demographic data (age, sex and life style), preoperative risk factors included cardiac disease, diabetes mellitus, hypertension, cerebrovascular disease, hypercholesterolemia, renal impairment, smoking history, body weight (using the body mass index), preoperative evaluation of PAD with complete medical history general examination vascular examination (palpable pulsation ankle pressure (AP) ankle brachial index (ABI) and duplex imaging or computed tomography angiography (CTA) for lesion evaluation of (site of lesion (common femoral + proximal or distal lesion) type of lesion (stenosis or occlusion), operative procedures perioperative complications, and follow up were recorded for each patient. 


\section{Revascularization procedures}

\section{Consent}

Patients after signing informed consent will proceed for hybrid repair (CFA endartrectomy and endovascular therapy), possible complication from the procedure and what are the alternatives.

\section{Procedure description}

All procedures were performed under local or regional (spinal and epidural) anesthesia in a hybrid operating room equipped with a mobile digital angiographic system (C-arm, Philips BV Pulsera ; Philips Medical Systems, Eindhoven, the Netherlands). The hybrid procedure under study was a planned one stage intervention including CFA Endarterectomy as a fixed step in all cases and an inflow or outflow endovascular revascularization step.

\section{CFA Endarterectomy step.}

We started our procedure by making a vertical inguinal incision.The common femoral artery was exposed from the circumflex femoral branches extended to the first $2 \mathrm{~cm}$ of the SFA and /or DFA.

Anticoagulation protocols were administration of unfractionated heparin at a dose of 70 to 100 units per kilogram of body weight as loading dose

External control of proximal common femoral artery and distal SFA and DFA was archived.

The common femoral artery was opened longitudinally toward the superficial femoral artery or profundafemoris artery.

The plaque was peeled away from the vessel wall in the direction of the arteriotomy, and removed with or without profundaplasty.
Suture tacking of the plaque to the posterior part of the adventitia were placed at the distal end of the Endarterectomy site to avoid plaque lifting, dissection, or thrombosis. The endarterectomized luminal surface was carefully inspected to check for a debris-free surface area.

Patch angioplasty was performed using saphenous vein in all cases.

\section{Endovascular revascularization step}

Concomitant EVT for iliac or femoropopliteal lesions was performed.

The patch was punctured and the endoluminal step performed under continuous blood flow and fluoroscopy guding. A $6 \mathrm{~F}$ to $7 \mathrm{~F}$ sheath was inserted over the wire; retrograde for inflow lesions and antegrade to complete the outflow recanalization.

Crossing the lesion was achieved using a combination of guidewires (0.035 Radifocus, standard/stiff type [Terumo Medical, Somerset, NJ, USA]; V-18 [Boston Scientific, Marlborough, MA, USA]; 0.018 Glidewire Advantage [Terumo]; and $5 \mathrm{~F}$ guiding catheters

All inflow lesions were managed by plain balloon angioplasty (PBA) with selective bare metal stenting for residual stenosis through retrograde access.

Outflow lesions were treated by PBA, regardless of lesion severity, and stenting was reserved for flow limiting dissections or residual stenosis $>30 \%$.

Balloon and stent diameters ranged from 7 to $9 \mathrm{~mm}$ for inflow arteries and from 5 to $6 \mathrm{~mm}$ for the femoropopliteal segment, and lengths ranged from 40 to $80 \mathrm{~mm}$ in iliac vessels and from 80 to $200 \mathrm{~mm}$ in the outflow arteries. 
The balloons used were Admiral Xtreme (Medtronic) and the stents were either balloon expandable (Visi-Pro; Medtronic) or self expandable (EverFlex [Medtronic]; E-Luminexx [Bard Peripheral Vascular, Tempe, AZ, USA]).

Completion angiography was always performed to check for residual stenosis or possible peripheral embolization. The sheath was removed and sutures were placed at the puncture site.

Aspirin (100 mg/day) and/or clopidgrel (75 mg/day) as well as cilostazol (100-200 $\mathrm{mg}$ /day) were orally administered after the procedure, and were continued as long as side effects did not occur.

\section{Follow up}

- Every patient will have visits every week till first month, then every 3 months till 1 years.
- Hemodynamic improvement was assessed by ankle brachial pressure index (ABI), performed before and after the procedure and every 3-4 months

- Evaluation made by CTA or duplex ultrasonography to determine 1ry and 2ndry patency

\section{Definations and endpoints:}

Technical success of CFE was determined by intraoperative angiography demonstrating less than $20 \%$ residual stenosis was considered technically successful.

Hemodynamic success was defined as an increase in $\mathrm{ABI}$ more than 0.10

Clinical success was defined according to the American Heart Association (AHA) classification (Table I).

Table I. American Heart Association guidelines for clinical improvement

\begin{tabular}{|c|l|} 
Grade & \multicolumn{1}{|c|}{ Clinical description } \\
$\mathbf{3}$ & Markedly improved; ABI 0.9 and no ischemic symptoms \\
$\mathbf{2}$ & 2 Moderately improved; ABI increase _ 0.1 but not normal, and increase by one category \\
$\mathbf{1}$ & Minimally improved; ABI increase 0.1 but not normal, or increase by one category \\
$\mathbf{0}$ & No change \\
$\mathbf{1}$ & Mildly worse; no category decrease or ABI increase Less than 0.1 \\
$\mathbf{2}$ & Moderately worse; one category worse or unexpected minor amputation \\
$\mathbf{3}$ & Markedly worse; more than one category worse or unexpected major amputation \\
\hline
\end{tabular}

Primary outcome

Primary patency was defined as patency of the CFA without evidence of restenosis or need for reintervention(in the 1 st 3 months without reintervention)

Primary-assisted patency was defined as a patent CFA that needed at least one reintervention for recurrent stenosis.

\section{Secondary outcome}

Limb salvage (Free from major amputation in 1st year)

\section{Statistical analysis.}

Continuous variables were reported as mean, standard deviation, and median, range. Categorical data were given as frequencies. Survival was evaluated by means of Kaplan- Meier analysis, comparison between groups regarding survival using log rank test. A P value $<0.05$ was considered statistically significant. Comparisons between groups were performed using a two-tailed $t$ test and one way anova for continuous variables and $\mathrm{X} 2$ test or fisher's exact test for categorical data. The statistical analysis was performed with SPSS 23 (SPSS Inc., Chicago, IL, USA). 
Results:

\section{Patients Characteristics}

A total of (30) patients underwent Hybrid technique CFE with proximal or distal angioplasty was done. Table I show the population consisted of

Table 1. Show patient Demographic data

\begin{tabular}{|l|c|c|}
\hline Age & No & $\%$ \\
Female & $58(44-79)$ & 16.5 \\
\hline
\end{tabular}

Table 2. Show Patient comorbidities

\begin{tabular}{|l|r}
\hline DM & N \\
HTN & 2 \\
Smoking & 2 \\
Hyerlipedimia & 1 \\
Obesity & 13 \\
IHD & 12 \\
CVD & 2 \\
CKD & 2 \\
\hline
\end{tabular}

\begin{tabular}{|c|c|} 
No & $\%$ \\
25 & 83.5 \\
24 & 80 \\
19 & 63.5 \\
13 & 43.5 \\
12 & 40 \\
20 & 66.5 \\
2 & 6.5 \\
0 & 0 \\
\hline
\end{tabular}

\section{Clinical presentation}

All patients had CLI, (46.5\%) of them presented with rest pain and (53.5\%) presented with tissue loss with association of variable degree of infection in $(43.5 \%)$ of them. On examination
(25) men and (5) women with a mean age of 58 years (range 44-79 years).

Table II; show the concomitant risk factors of the study population.

Table 3. Clinical presentation

\begin{tabular}{|ccc|}
\hline Rest pain & No & $\%$ \\
Tissue loss & 14 & 46.5 \\
Infection & 16 & 53.5 \\
Intact Femoral pulse & 13 & 43.5 \\
\hline
\end{tabular}

\section{Pre-operative assessment:}

We reported $100 \%$ CFA lesion in which only $6(20 \%)$ have tight stenosis and $24(80 \%)$ have femoral plus was detected with same power compared to other site in 7 patients and with weak power in 2 patients and absent in other patients (Table 3)

total occlusion. The associated other lesion are proximal iliac lesion in $10 \%$ of patients, SFA in $33 \%$, tibial in $30 \%$ and combined iliac and SFA in $27 \%$. Table 4,5

Table 4. Type of femoral lesion

\begin{tabular}{|lcc|}
\hline & No & $\%$ \\
Stenosis & 6 & 20 \\
Occlusion & 24 & 80 \\
\hline
\end{tabular}

\section{Table 5.: Distribution of associated other lesion}

\begin{tabular}{|lcc|}
\hline & No & $\%$ \\
Iliac & 3 & 10 \\
SFA & 10 & 33 \\
Tibial & 9 & 30 \\
Iliac + SFA & 8 & 27 \\
\hline
\end{tabular}

Table 6 shows The type of other lesions is stenosis which affect $18(60 \%)$ patients and total occlusion which affects $12(40 \%)$. 
Table 6: Type of associated lesion

\begin{tabular}{|lcc|}
\hline & No & $\%$ \\
Stenosis & 18 & 60 \\
Occlusion & 12 & 40 \\
\hline
\end{tabular}

\section{Procedural Details}

The procedures were performed under local anesthesia with conscious sedation in 1 (3.3\%) or regional (spinal and epidural) in 29 patients $(96.6 \%)$.

\section{1. $\underline{\mathrm{CFE}}$}

CFA Endarterectomy was performed in $100 \%$ of patients

2. Proximal lesion

Concomitant in flow or proximal (iliac) lesion 11(36\%) treated by endovascular thereby by balloon dilation in 9 patients $(81.8 \%)$ and selective stenting in $2(18.1 \%)$ through retrograde access .

\section{Distal lesion}

The out flow or distal lesion femropopletial are 10 patient (33.3\%) and tibial 9 patients $(30 \%)$.The femoropopltial lesion treated by selective stenting of 2 patients (10.5\%) and balloon dilation in $8(42.1 \%)$ and tibial 9 patients $(47.3 \%)$ treated by balloon dilation
We made balloon dilatation in $90 \%$ of distal lesions while stent was used only in $2(10 \%)$ of patients.

\section{Outcome}

\section{- Intraoperative:}

Technical success was achieved in $100 \%$ of the procedures. The intraoperative complication rate was $(6.66 \%)$ : in two patients the first intraoperative angiography showed a dissection of the SFA, and another patient underwent thromboembolectomy due to an intraoperative peripheral embolization detected by the first angiography.

\section{- Post-operative:}

Hemodynamic success was achieved in 29 patients (96\%). The median ABI improved significantly, rising from $0.3(0.2-0.4)$ preoperatively to $0.7(0.4-0.9)$ postoperatively. Table

\begin{tabular}{|c|c|}
\hline $\begin{array}{l}\text { AP pre } \\
\text { ABI pre } \\
\text { ABI post }\end{array}$ & $\begin{array}{c}\text { Median (min-Max) } \\
42(30-55) \\
0.3(0.2-0.4) \\
0.7(0.4-0.9)\end{array}$ \\
\hline Clinical success was achieved in 29 & 2, and $13.5 \%(4 / 30)$ grade 1 , according to the \\
\hline
\end{tabular}
having grade 3 improvement, 56.5\% (17/30) grade

Table 8 shows the distribution of clinical success score.

\begin{tabular}{|lcc|}
\hline & No & $\%$ \\
0 & 1 & 3.5 \\
1 & 4 & 13.5 \\
2 & 17 & 56.5 \\
3 & 8 & 26.5 \\
\hline
\end{tabular}

Post-operative complication was reported in $2(6.6 \%)$ of patients, one with small incisional haematoma and the other with superficial wound infection; both managed conservatively. 


\section{Follow-up:}

With the mean follow up 18 months (range 1-26) we reported post-operative $\mathrm{ABI} 0.7 \pm 0.17$ which achieve statistical significance between pre and post $\mathrm{ABI} \mathrm{P}=0.0001$.

Comparing the pre-operative clinical character with the improvement in Postoperative ABI we reported that patient with palpable CFA have more improvement than those without intact CFA pulsation with statistical significance $\mathrm{P}=0.012$. Otherwise, we found no difference in the outcome whether the CFA was stenosed or totally occluded with $\mathrm{p}=0.7$.

Similar to the CFA type of lesion we reported no statistical significance regarding the outcome and the associated proximal or distal lesion type $\mathrm{P}=0.8$.

On the other hand, the site of associated proximal or distal lesion affected the outcome significantly $\mathrm{P}=0.001$ in which the tibial lesion had

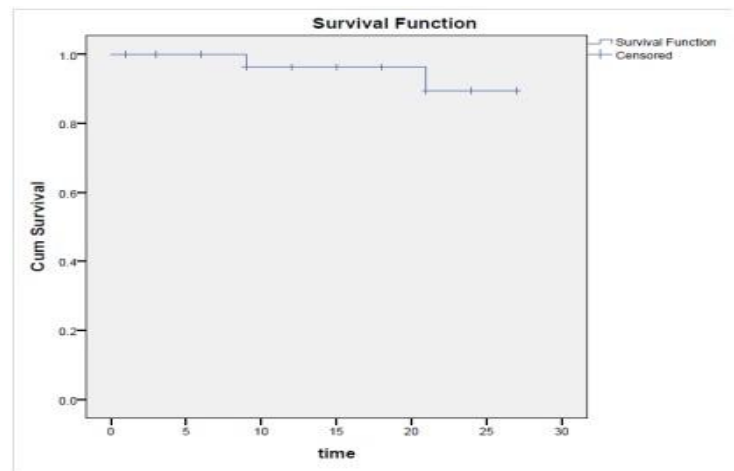

Regarding the limb salvage we had only one patient who underwent BKA at 10 months and we the best outcome while the combined Iliac and SFA have the worst outcome.

The preoperative clinical diagnosis whether rest pain or tissue necrosis doesn't affect outcome. Surprisingly, also the presence of infection doesn't affect outcome.

\section{Late outcome:}

We assess late outcome in terms of CFA patency and limb salvage at 3, 6, 9, 12, 18, 24 months.

We reported patency of CFA $100 \%$ at 3, 6 months and $96 \%$ at $9,12,18$ months and $88 \%$ at 24 months in which only one patient had CFA reocclusion at 9 months follow up and another one at 21 month follow up managed conservatively as they had no symptoms Showed in KM curve 1.

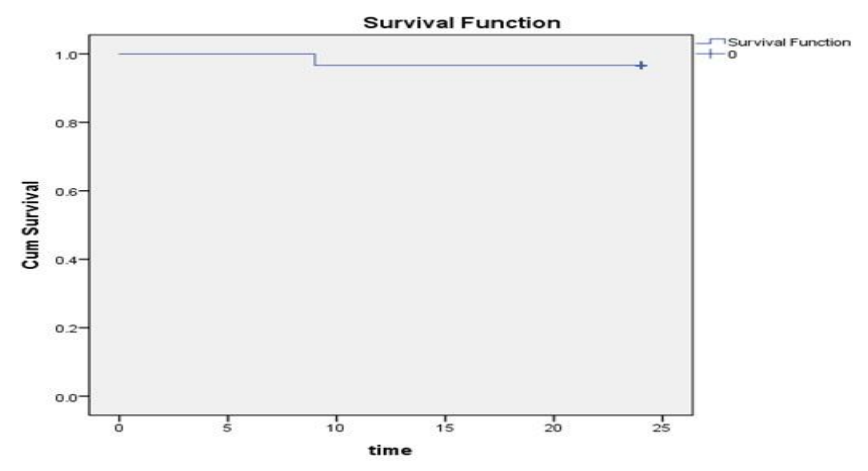




\section{Discussions}

CLI patients usually have multilevel PAD and significant combined disease, and the management is a challenge to vascular specialist. Endovascular intervention is an attractive alternative to open surgery in the treatment of patient with multilevel PAD, especially in highrisk patients.(4)

However, endovascular procedures of CFA often fails and may show suboptimal results because the atherosclerotic lesion in this area usually is heavily calcified and bulky (5) Many interventionalists avoid CFA stenting because deployment across a high mobility joint area may result in a more extensive neointimal hyperplasia. Therefore, CFA endarterectomy and patch angioplasty remain to be the gold standard treatment for CFA occlusive disease simultaneous with profundaplasty to optimise the runoff, and to provide an access point for inflow and/or outflow endovascular treatment (6)

The greatest advantage of endarterectomy is its capability of fully debulking the atherosclerotic intima with or without patch augmentation to obtain the greatest diameter (7)

Initial reports of hybrid technique date from the 1970s (8) Since then, most studies have investigated CFA endarterectomy or femorofemoral bypass combined with inflow endovascular therapy. They have reported comparable long term results to open surgical procedures, with lower or equal morbidity and mortality rates $(\mathbf{9})$

Hybrid open and endovascular procedure can be performed on the same setting or as a staged procedure. Staged approach, compared to simultaneous hybrid procedure, is associated with longer hospital stay, high cost, groin wound problems, and additive cardiac risk with multiple procedures (10)

Because simultaneous hybrid procedure is performed under fluoroscopy, results of the treatment can be improved by immediately verifying the procedural success.

CLI patients are frequently elderly and frail with high mortality. Taking this into account, the simultaneous hybrid procedure is a feasible and efficacious treatment for CLI patients with multilevel high-risk atherosclerotic disease(10)

Recently, hybrid common femoral endarterectomy and EVT for multilevel occlusive artery disease has been established. Simultaneous CFE plus EVT for iliac or superficial femoral artery lesions is suggested to represent a valid alternative procedure to open aortoiliofemoral or infrainguinal bypass grafting (11)

The current prospective study aimed to assess the outcomes of common femoral endarterectomy combined with an inflow and outflow endovascular revascularization procedure as an alternative for high risk patients with CLTI. Included cases were treated rather homogenously under local or regional anaesthesia.

The current study included all consecutive patients who presented with CLTI due to multilevel arterial disease involving the CFA

Femoral Endarterectomy was performed in all cases in the current cohort with combined iliac and SFA revascularization in $27 \%$ of patients and with separate iliac in $10 \%$ and outflow in $63 \%$ of cases (SFA 33\% and tibial 30\%).

Matsagkas et al. reported that CFA Endarterectomy was combined with distal endoluminal procedures in $52.2 \%$ of limbs, with 
proximal endoluminal procedures in $29.5 \%$ and with both proximal and distal endoluminal procedures in only $18 \%(\mathbf{1 2})$

This study reported a $100 \%$ technical success and 96\% hemodynamic and clinical success in the current series. And the 2-year patency was $88 \%$ These results were in agreement with those reported by Matsagkas et al., in a series of 37 patients (44 limbs) using one-stage hybrid technique with technical and hemodynamic success rates of 96.6 and 100\%, respectively(12)

In accordance to this cohort results; Antoniou et al. reported technical and hemodynamic success rates of 100 and $95 \%$, respectively, from 60 patients who underwent 61 single-step hybrid procedures(13)

Additionally, Zou et al. and Jung et al. Reported technical success rates of 98 and 100\%, respectively, following the same management protocol (14)(15)

Nelson et al.13 reported their initial experience of combined $\mathrm{CFE}$ and external iliac artery stenting; technical and hemodynamic success rates were both $100 \%$; the 1-year primary and assisted primary patency rate were $84 \%$ and 97\%, respectively(11)

Cotroneo et al. reported the 2-year results of hybrid CFE and EVT, including iliac artery and/or superficial femoral, profunda femoris, and popliteal endovascular procedures. The technical success rate was $100 \%$ and the 2-year primary and assisted primary patency rates were $79 \%$ and $86 \%$, respectively (16)

Jung et al.studied 38 consecutive patients with CLI (43 limbs) with multilevel peripheral arterial disease who were treated by one-stage hybrid technique which combined CFA endarterectomy and additive interventional procedure. The primary and secondary patency rates at 24 months were 67.3 and $72.1 \%$, respectively. The limb salvage rate was $95.3 \%$ (15)

On the contrary, Matsagkas et al. reported higher 2-year primary and secondary patency rates of 93.2 and $95.5 \%$, respectively. This higher patency rate could be attributed to the different patient characteristics as most of their patients $(52.2 \%)$ presented with claudication.

In the current study, the primary patency rate was lower in diabetic than nondiabetic patients and occlusion than stenosis of CFA.

This is in accordance with Spanos et al. who in a retrospective analysis from a single center of 132 patients who underwent hybrid procedures for the management of multisegmental chronic peripheral arterial disease reported that the hazard ratio for primary patency failure was 1.94 times higher in diabetic patients $(\mathrm{P}=0.029)$ (17)

Supporting this study results, Nishibe, $\mathrm{T}$ et al did not find any predictors of decreased primary patency. There were no statistically significant differences in primary patency rates between patients with claudication versus CLI, between diabetic versus nondiabetic patients, between patients on hemodialysis versus nonhemodialysis, and between patients undergoing isolated CFE and hybrid CFE plus EVT(18)

CFE exhibited a high technical success rate of $85-100 \%$ as well as low morbidity and mortality rates of $6.6-22 \%$ and $0-2 \%$, respectively (19)

This series showed low rate of perioperative morbidity, only 2 patients suffering from complications; one with access site 
hematoma and the other with superficial surgical wound infection.

Recently, Siracuse et al. collected a total of 1513 patients undergoing elective CFE and showed that the 30-day postoperative mortality rate was $1.5 \%$. Postoperative morbidities included cardiac $(1.0 \%)$, pulmonary $(1.9 \%)$, renal $(0.4 \%)$, urinary tract infection (1.7\%), thromboembolic (0.5\%), neurologic (0.4\%), sepsis (2.7\%), superficial (6.3\%), and deep surgical site complications $(2.0 \%)$. At least one complication, either major or minor, was seen in $7.9 \%$ of the patients. We also achieved no major perioperative complication (death or amputation) and a low rate of local complications (11\%), including surgical wound infection, bleeding, and anastomotic false aneurysm(20)

A series of literatures investigated the role of endovascular techniques in management of CFA lesions.

Bonvini et al. retrospectively analyzed the outcomes of 360 consecutive endovascular interventions for common femoral artery lesions in 321 patients. Balloon angioplasty and provisional stenting were associated with a high success rate of $93 \%$ and low rates of in-hospital major and minor complications of $1.4 \%$ and $5 \%$, respectively. However, the 1-year restenosis and target lesion revascularization rates were disappointing, at $27.6 \%$ and $19.9 \%$, respectively (21)

Soga et al. reported that, in 214 common femoral artery lesions treated by EVT, the 1- and 5 -year primary patency rates were not promising, at $73.5 \%$ and $56.2 \%$, respectively. At the current time, EVT for common femoral artery lesions does not appear to be feasible, and CFE remains the preferred treatment for common femoral artery lesions in our practice (22)

Mehta and his colleagues reported the effect of DEB angioplasty in CFA occlusive disease; the primary 1-year patency rate of DEB angioplasty for the CFA was $75.0 \%$, with $97.5 \% 1$ year secondary patency rate, which was similar to that of plain PTA for CFA disease in the literature ranging from $70 \%$ to $84 \%$ 1-year patency rate. (23)

On the other hand, CFA stenting is a debatable issue, some authors are against stenting as it is liable for fracture and obscure the access for angioplasty. Others reported their experience with the use of stenting in CFA lesions.

Stricker and Jacomella analyzed 33 angioplasties in 27 patients with stent implantation in the CFA bifurcation. PP rates were $87 \%$ at 1 year, $86 \%$ at 2.5 years, and $83 \%$ at 3 years. All patients received one short stent after angioplasty. Intimal hyperplasia appeared in five patients with restenosis, and a stent fracture occurred in one patient after 10 months (Stricker $\mathrm{H}$ et al 2004)

Azéma et al treated 36 patients in a 2-year period. The indication for endovascular revascularization of the CFA included IC in 25 (69.4\%). At 1 year, primary and secondary sustained clinical improvements were $80 \%$ and 90\%. The authors reported an in-stent restenosis rate of $20 \%$, with one observed stent fracture.(24)

Emerging endovascular techniques such as laser angioplasty, atherectomy, cryoplasty, as well as bio absorbable stent placement have been applied to treating common femoral artery lesions. Also Linni and contributers initiated a randomized, controlled, single center, open-label trial to compare clinical and hemodynamic outcomes in 
patients undergoing treatment of the CFA by bio absorbable stent implantation or by CFE. The primary end points were technical success, hemodynamic improvement, clinical improvement, patency, limb salvage, and survival. Technical success rates were $100 \%$ for open surgery and $97.5 \%$ for ET. Hemodynamic improvement considering the postoperative $\mathrm{ABI}$ was comparable between the two groups (P 1/4.38). The 30-day PP rates were $92.5 \%$ for ET and $100 \%$ for CFE patients ( $\mathrm{P} 1 / 4.038)$. At 1 year, the $\mathrm{PP}$ and $\mathrm{SP}$ rates were $80 \%$ vs $100 \%$ for ET patients (P 1/4.007) and $84 \%$ vs $100 \%$ (P $1 / 4.01$ ) for CFE patients. Survival rates were $88 \%$ for ET vs $90 \%$ for CFE patients (P 1/4.51). The authors concluded that stent implantation has only limited eligibility for CFA stenosis and is not an option for CFA occlusion.(25)

The of our study Limitations First, the relatively small number of patients, the relatively short follow-up period, and the low incidence of adverse events may affect the reliability of our results. Finally, the lack of control or comparison group data, including open surgical bypass data, may prevent any conclusive analysis of CFE.

\section{CONCLUSIONS}

In conclusion, we corroborate the concept that CFE should remain the treatment of first choice of CFA occlusive disease as it is safe, effective, and durable procedure for common femoral artery disease, and the simultaneous hybrid CFA endarterectomy and endovascular therapy for multilevel occlusive artery disease can provide an immediate satisfactory results, durable midterm patency, and also less invasive therapeutic option in high-risk patients. The hybrid CFE plus EVT can be a valid alternative to open surgical bypass.
These observations stress that CFE plays a vital role in the management of PAD even in the endovascular era.

\section{References}

1. Stoner MC, Calligaro KD, Chaer RA, Dietzek AM, Farber A, Guzman RJ, et al. Reporting standards of the Society for Vascular Surgery for endovascular treatment of chronic lower extremity peripheral artery disease. J Vasc Surg. 2016;64(1):e1-21.

2. Aboyans V, Ricco JB, Bartelink M, Björck M, Brodmann M, Cohnert T, et al.; ESC Scientific Document Group. 2017 ESC Guidelines on the Diagnosis and Treatment of Peripheral Arterial Diseases, in collaboration with the European Society for Vascular Surgery (ESVS): Document covering atherosclerotic disease of extracranial carotid and vertebral, mesenteric, renal, upper and lower extremity arteriesEndorsed by: the European Stroke Organization (ESO)The Task Force for the Diagnosis and Treatment of Peripheral Arterial Diseases of the European Society of Cardiology (ESC) and of the European Society for Vascular Surgery (ESVS). Eur Heart J. 2018;39(9):763-816.

3. Cardon A, Aillet S, Jarno P, Bensalah K, Le Du J, Idrissi A, et al. Endartériectomie du trépied fémoral: résultats à long terme et analyse des facteurs d'échec [Endarteriectomy of the femoral tripod: long-term results and analysis of failure factors]. Ann Chir. 2001;126(8):777-82.

4. Norgren, L., Hiatt, W. R., Dormandy, J. A., Nehler, M. R., Harris, K. A., Fowkes, F. G., \& TASC II Working Group (2007). Inter-Society Consensus for the Management of Peripheral Arterial Disease (TASC II). Journal of vascular surgery, 45 Suppl S, S5-S67.

5. Johnston KW, Rae M, Hogg-Johnston SA, Colapinto RF, Walker PM, Baird RJ, et al. 
5-year results of a prospective study of percutaneous transluminal angioplasty. Ann Surg. 1987;206(4):403-13.

6. Kang JL, Patel VI, Conrad MF, Lamuraglia GM, Chung TK, Cambria RP. Common femoral artery occlusive disease: contemporary results following surgical endarterectomy. J Vasc Surg. 2008;48(4):872-7.

7. Diehm C, Schuster A, Allenberg JR, Darius H, Haberl R, Lange S, et al. High prevalence of peripheral arterial disease and co-morbidity in 6880 primary care patients: cross-sectional study. Atherosclerosis. 2004;172(1):95-105.

8. Porter JM, Eidemiller LR, Dotter CT, Rösch J, Vetto RM. Combined arterial dilatation and femorofemoral bypass for limb salvage. Surg Gynecol Obstet. 1973;137(3):409-12.

9. Aho, P. S., \& Venermo, M (2012). Hybrid procedures as a novel technique in the treatment of critical limb ischemia. Scandinavian journal of surgery : SJS : official organ for the Finnish Surgical Society and the Scandinavian Surgical Society, 101(2), 107-113.

10. Patel SD, Donati T, Zayed H. Hybrid revascularization of complex multilevel disease: a paradigm shift in critical limb ischemia treatment. J Cardiovasc Surg (Torino). 2014;55(5):613-23.

11. Nelson PR, Powell RJ, Schermerhorn ML, Fillinger MF, Zwolak RM, Walsh DB, et al. Early results of external iliac artery stenting combined with common femoral artery endarterectomy. J Vasc Surg. 2002;35(6):1107-13.

12. Matsagkas M, Kouvelos G, Arnaoutoglou E, Papa N, Labropoulos N, Tassiopoulos A. Hybrid procedures for patients with critical limb ischemia and severe common femoral artery atherosclerosis. Ann Vasc Surg. 2011;25(8):1063-9.

13. Antoniou, G. A., Sfyroeras, G. S., Karathanos, C., Achouhan, H., Koutsias,
S., Vretzakis, G., \& Giannoukas, A. D (2009). Hybrid endovascular and open treatment of severe multilevel lower extremity arterial disease. European journal of vascular and endovascular surgery : the official journal of the European Society for Vascular Surgery, 38(5), 616-622.

14. Zou J, Xia Y, Yang H, Ma H, Zhang X. Hybrid endarterectomy and endovascular therapy in multilevel lower extremity arterial disease involving the femoral artery bifurcation. Int Surg. 2012;97(1):56-64.

15. Jung HJ, Lee SC, Kim KY, Lee SS. Simultaneous Hybrid Operation Common Femoral Endarterectomy and Endovascular Treatment in Multilevel Peripheral Arterial Disease with Critical Limb Ischemia. Indian J Surg. 2018;80(2):140-5.

16. Cotroneo AR, Iezzi R, Marano G, Fonio P, Nessi F, Gandini G. Hybrid therapy in patients with complex peripheral multifocal steno-obstructive vascular disease: two-year results. Cardiovasc Intervent Radiol. 2007;30(3):355-61.

17. Spanos, K., Antoniou, G. A., Saleptsis, V., Athanasoulas, A., Drakou, A., Koutsias, S., \& Giannoukas, A. D (2017). Hybrid procedures for chronic lower limb ischemia: what determines the outcome? International angiology : a journal of the International Union of Angiology, 36(2), 174-181.

18. Nishibe $T$, Maruno $K$, Iwahori $A$, Fujiyoshi T, Suzuki S, Takahashi S, et al. The Role of Common Femoral Artery Endarterectomy in the Endovascular Era. Ann Vasc Surg. 2015;29(8):1501-7.

19. Ballotta E, Gruppo M, Mazzalai F, Da Giau G. Common femoral artery endarterectomy for occlusive disease: an 8-year single-center prospective study. Surgery. 2010;147(2):268-74. 
20. Siracuse JJ, Huang ZS, Gill HL, Parrack I, Schneider DB, Connolly $\mathrm{PH}$, et al. Defining risks and predicting adverse events after lower extremity bypass for critical limb ischemia. Vasc Health Risk Manag. 2014;10:367-74.

21. Bonvini RF, Rastan A, Sixt S, Noory E, Schwarz T, Frank U, et al. Endovascular treatment of common femoral artery disease: medium-term outcomes of 360 consecutive procedures. J Am Coll Cardiol. 2011;58(8):792-8.

22. Soga Y, Tomoi Y, Sato K, Iida O, Yokoi H. Clinical outcome after endovascular treatment for isolated common femoral and popliteal artery disease. Cardiovasc Interv Ther. 2013;28(3):250-7.

23. Mehta M, Zhou Y, Paty PS, Teymouri M, Jafree K, Bakhtawar H, et al. Percutaneous common femoral artery interventions using angioplasty, atherectomy, and stenting. J Vasc Surg. 2016;64(2):369-79.

24. Azéma, L., Davaine, J. M., Guyomarch, B., Chaillou, P., Costargent, A., Patra, P., \& Gouëffic, Y (2011). Endovascular repair of common femoral artery and concomitant arterial lesions. European journal of vascular and endovascular surgery : the official journal of the European Society for Vascular Surgery, 41(6), 787-793.

25. Linni, K., Ugurluoglu, A., Hitzl, W., Aspalter, M., \& Hölzenbein, T (2014). Bioabsorbable stent implantation vs. common femoral artery endarterectomy: early results of a randomized trial. Journal of endovascular therapy : an official journal of the International Society of Endovascular Specialists, 21(4), 493-502. 